\title{
A Fuzzy Reasoning Approach for Assessing Morningness of Individuals Using Reduced Version of Morningness-Eveningness Questionnaire
}

\author{
Debasish Majumder $^{a}$, Subhashis Sahu ${ }^{b}$ and Animesh Biswas ${ }^{c *}$ \\ ${ }^{a}$ Department of Mathematics, JIS College of Engineering, Kalyani-741235, India \\ ${ }^{b}$ Department of Physiology, University of Kalyani, Kalyani-741235, India \\ ${ }^{c}$ Department of Mathematics, University of Kalyani, Kalyani-741235, India
}

Received 4 May 2016

Accepted 31 October 2016

\begin{abstract}
In this article assessment of morningness of individuals has been performed using fuzzy reasoning approach. The responses are quantified using fuzzy numbers. Based on experts' opinion a fuzzy rule-base is prepared. The model is validated by considering responses of some students, selected randomly, and assessing their degree of morningness. The achieved results are compared with that of existing classical method. Results show that proposed approach outperforms the existing classical approaches by capturing the inherent ambiguity and vagueness of morningness study.
\end{abstract}

Keywords: Reduced version of Morningness-Eveningness Questionnaire; preference scale; fuzzy numbers; fuzzy reasoning; artificial intelligence.

\section{Introduction}

Fuzzy logic is a precise logic of imprecision and approximate reasoning ${ }^{1,2}$. One of the most important contributions of fuzzy logic is its high power of precision which enable it to serve as a co-intensive model of reality, especially in human-centric fields such as economics, law, linguistics and psychology ${ }^{3-5}$. Most of human knowledge is reflected using natural language. Natural languages are pervasively imprecise in the sense that in natural language almost everything is a matter of degree.

The proficiency of fuzzy sets in formalizing mathematically imprecise valuations and the ability of mathematical computations with them are exploited in designing questionnaires used to assess many domains

*Corresponding author. E-mail: abiswaskln@rediffmail.com. of psychology-related issues, such as perceptions, opinions, emotional states, etc. In contrast to the conventional integer scale based questionnaires, for which responses to questions are constrained to choosing one within a list of prefixed labels, the flexibility and expressiveness questionnaires based on the fuzzy rating scale allow us to properly describe the answers to many questions involving psychological measurement ${ }^{6}$. Over the last few years, several comparative studies have been conducted to verify statistically some immediate intuitive benefits obtained by using the fuzzy rating method in designing questionnaires to collect human ratings, valuations, perceptions, judgments, etc ${ }^{7,8}$.

\subsection{Morningness}

Individual differences in circadian rhythm, popularly known as morning-evening orientation of individuals, 
affect our physiological and psychological functioning, not only in health, but also in disease ${ }^{9}$. Several studies have shown that there exists a strong relationship between physiological measures and morningness, as well as differences in phase position between the two extreme types ${ }^{10,11}$. Several research works have been performed to investigate the morning-evening orientation of individuals in order to identify the time of best performance in different fields like sports ${ }^{12}$, education $^{13}$, shift-work ${ }^{14}$, etc. Though the circadian period may be determined experimentally in human beings, most feasibly through the forced desynchronized protocol $^{15}$, it is costly, intrusive, and time-consuming, and thus not a feasible option for large-scale human phenotype. A more practical proxy for such studies is diurnal preference or chronotype, a self-reported questionnaire-based instrument that yields an integer score (crisp) on a scale ranging between extreme morning preference and extreme evening preference. Among the several types questionnaires, like Morningness-Eveningness Questionnaire (MEQ $)^{16}$, Circadian Typology Questionnaire (CTQ) $)^{17}$, Composite Morningness Questionnaire $(\mathrm{CMQ})^{18}$, etc., used to measure circadian rhythms and chronotypes, reduced version of MEQ (rMEQ) ${ }^{19}$ has proven to be a quick and reliable measure, with adequate inter-item correlation and validity ${ }^{20}$.

\subsection{Fuzzy logic in morningness studies}

The questionnaires, used in assessing morningness of individuals, frequently involve linguistic terms whose meanings are vague/fuzzy in nature. Moreover, the items, asked to fill up in the questionnaire, may have the complex nonlinear relationship with individual differences in circadian rhythm. Conventional statistical approaches $^{11,12,13}$ (classical approaches) are unable to capture the vagueness/fuzziness associated with morningness study and hence may not provide the satisfactory result. Milia, et $a l^{21}$, emphasize the methodological limitations that exist in morningness study.

In order to overcome these limitations, a very few study of morningness using fuzzy logic have been made in the recent past. Biswas et l. $^{22}$, conducted the morningness study by developing a fuzzy expert system and an Adaptive Neuro Fuzzy Inference Systems (ANFIS) with the aim of capturing the impreciseness or vagueness associated with the frequently used linguistic terms in rMEQ. In 2014, some research works was performed to generate different types of hybrid fuzzy inference systems like Adaptive Neuro Fuzzy Inference Systems (ANFIS) ${ }^{23}$, Genetic Algorithm Based Mamdani Fuzzy Inference System (GAMFIS) ${ }^{24}$, for assessing morningness of people using rMEQ. Despite the advantages of these hybrid fuzzy systems, the conventional integer scoring scale of rMEQ has several weaknesses.

\subsection{Motivation of the present study}

The rMEQ, the most frequently used questionnaire in morningness study, comprises five questions based on the original version of the MEQ. Questions 1, 3 and 4 ask subjects to indicate the time they prefer to get up, the time they prefer to go to bed and the time of the day when they feel best. Question 2 is related to the degree of tiredness perceived in the first half hour after waking up in the morning. Finally, in question 5, subjects are asked to indicate their morningness or eveningness preferences. All the questions are associated with a set of pre-fixed categories which are usually coded by means of integer numbers from a scale usually ranging from 1 to 4 , or from 1 to 5 , or from 1 to 6 . The responses of these questions of $\mathrm{rMEQ}$ are traditionally viewed as values of linguistic variables, but still encoded by means of integer numbers, in spite of the many concerns associated with such an encoding. This study addresses some neglected issues in morningness research using rMEQ.

- Firstly, in rMEQ, the first, third, and fourth questions are related to the time of preference of an individual for performing his/her daily activities. Now, to give response to those questions, it is difficult for any individual to identify precisely a specific time of a day for those activities. As a result, they generally prefers to give the response of those questions either as an interval of time or in natural languages, like between 5:00 a.m. and 5:30 a.m., around 10:00 p.m., before 6:00 a.m., after 5:30 p.m., etc. The scoring was done on the range. Since, most of these responses may not indicate precise time of a day and sometimes those responses may also be in natural languages, vagueness and/fuzziness are inherent with those responses. Hence, at the time of assessing morningness if those responses are expressed by a precise (or crisp) score on a preference range may not be able to capture the vagueness and/uncertainties associated with those responses. 
- Secondly, the responses of first, third, and fourth questions in rMEQ, may not match with any particular option in the questionnaire rather may be in between any two consecutive options out of the available options of that particular question. For example, an individual may reach his/her "feeling best peak" between 7:30 a.m. and 8:30 a.m. which actually fall in between the first two options corresponding to the fourth question in rMEQ. As a result, either he/she will be bound to leave the fourth question without giving any response or he/she might choose only one option out of first two options. Eventually, in both cases, the study of morningness will be affected due to the lack of actual response.

- Thirdly, in rMEQ, second and fifth questions are related to an individual's own feelings and his/her self-assessment. When those questions are asked to an individual he will feel comfort to express his/her feelings and self-assessment either by using qualitative terms, viz., "tired", "fairly tired", "refreshed", "very refreshed" etc. or in natural languages like "definitely morning", "rather morning than evening", "rather morning than evening" etc. whose interpretation are not straightforward. It completely depends on human perception. However, human perception is not precise and may vary over time; one's intuitions and cognition of a concept highly depends on the context, domain knowledge, individual senses, etc. Hence, different individuals may perceive the same concept differently ${ }^{25,26}$. So, different individuals may use same qualitative descriptors to express different state of feelings; on the other hand, they may use different qualitative descriptors to express same state of feelings. Thus, natural languages are pervasively imprecise in the sense that in a natural language almost everything is a matter of degree. Imprecision of natural languages is an issue of central importance. Unfortunately, despite its importance, the issue of imprecision has not been studied earlier in the context of assessing morningness.

- Fourthly, the duration of "feeling best peak" may vary from person to person. For example, one individual may reach his/her "feeling best peak" between 10:30 a.m. and 11:30 a.m. whereas other may reach his/her "feeling best peak" between 10:30 a.m. and 12:30 p.m. In such cases, though both of them will choose the third option of the fourth question but the duration of "feeling best peak" for first individual is one hour whereas for second individual it is two hours and hence, quite naturally, these two different responses should have different impact on morning-evening orientation. But, unfortunately, in present rMEQ, both are treated equally and getting same score in preference scale which is quite unnatural.

- Lastly, the responses corresponding to each of the five questions of rMEQ might not have equal effect on morning-evening orientation and hence may have some non-linear relationship with morningness. As a result, total scores may not be an appropriate measure of circadian rhythms and chronotypes as multidimensional constructs. Thus, relationship of five items of rMEQ with morningness is important issue in the study of morningness. Unfortunately, this issue also remains ignored in the literatures of morningness study.

In artificial intelligence, the relation between fuzzy logic and natural languages is two-sided. On one side, fuzzy logic draws on natural languages in the formalism of the linguistic variables and the calculi of fuzzy ifthen rules. On the other side, being logic of imprecision and approximate reasoning, fuzzy logic has a greater contribution to the formalization of natural languages, especially in the realm of semantics. So it plays an essential role in mechanization of natural language understanding ${ }^{3}$.

In this paper, a novel preference scale based on fuzzy sets theory has been proposed. After collecting the responses from the respondents through questionnaire in the form of quantitative as well as qualitative manners, the preference scales based on fuzzy numbers are used to measure the responses. Since the fuzzy numbers are defined on some continuous universe of discourse, most of the time the scales are unbalanced in nature. Also the respondents are free to exert their responses without any bound stipulated by the questionnaire.

Moreover, a new methodology for assessing morningness of individuals has been developed using fuzzy reasoning approach (FRA). This method differs from the previous approaches viz., ANFIS and GAMFIS methods, because in both approaches crisp values are used for the input-output parameters of the fuzzy inference system (FIS); whereas in the proposed method fuzzy numbers are used not only to capture the inexactness associated with the data provided by the individuals but also to cope up with fuzzy terms associated with answers given by different individuals. 


\section{Methodological Development}

In the present study, a new methodology has been developed for assessing morningness of individuals on the basis of responses given to the rMEQ using FRA as described in the following sub-sections.

\subsection{Data collection}

In order to get better demonstration, a proper discussion on the items of the questionnaire as well as all details about the study need to be clarified before filling up the questionnaire by the individuals. The better understanding of all five questions of rMEQ will help an individual to correctly express his/her responses. For the present study, responses are collected from university students after discussing all the details about the study. In view of the motivation of the present study, responses of 10 individuals are taken into consideration for the demonstration of the proposed methodology as well as to illustrate how the proposed methodology can address those neglected issues in morningness study.

\subsection{Input parameters selection}

In the present study, input parameters are selected on the basis of items in rMEQ. In this context, Average Rising Time for Feeling Best $\left(X_{1}\right)$, Feelings within First Half an Hour after waken up in the morning $\left(X_{2}\right)$, Sleeping Time $\left(X_{3}\right)$, Feeling Best Time in a Day $\left(X_{4}\right)$ and Self-Assessment about Morningness $\left(X_{5}\right)$ are are considered as input parameters to measure the output parameter Morningness $(Z)$.

\subsection{Development of a fuzzy rule base}

A fuzzy rule base consists of a set of fuzzy if-then rules $^{27}$ which are used to capture the complex or nonlinear relationship between variables of different real life problems. These fuzzy rules can be derived by several ways such as data driven method, experts' judgment and knowledge acquisition. In this study, experts' judgment and knowledge acquisition techniquesare used to derive the fuzzy rules which are generally expressed in terms of qualitative descriptors of input and output variables. The qualitative descriptors are characterized by fuzzy sets those are derived by membership functions (MFs). Among the several types of MFs, linear type MFs is widely used due to its simple linear characteristic. However, trapezoidal type MFs can capture the property of all other types of linear MFs, viz., triangular MFs, Z-shaped MFs, S-shaped MFs, etc. From that view point, trapezoidal type MFs is used here to determine the different qualitative descriptors of input and output parameters.

\subsection{FRA for morningness assessment}

Morningness of an individual is generally assessed on the basis of his/her responses corresponding to the questions of different types of questionnaires. Since, it is difficult for an individual to provide answer to those questions in a precise manner due to the uncertainties involved in his/her responses to different questions or due to presence of some quantitatively immeasurable parameters in the morningness assessment process. The process of assessment thus frequently faces the circumstances where the data are imprecise and sometimes uncertain also. In such situation, it is conformable to consider the input values as a range of numerical values or fuzzy numbers or linguistic terms to express their responses. In this paper, trapezoidal fuzzy numbers (TFNs), as a general form of linear type fuzzy numbers, are used to capture responses of the individual. A TFN is expressed in the form $F=$ $\left\langle a_{1}, a_{2}, a_{3}, a_{4}\right\rangle$ with its membership function

$$
\mu_{F}(x)=\left\{\begin{array}{cc}
\frac{x-a_{1}}{a_{2}-a_{1}}, & a_{1} \leq x \leq a_{2} \\
1, & a_{2} \leq x \leq a_{3} \\
\frac{x-a_{4}}{a_{3}-a_{4}}, & a_{3} \leq x \leq a_{4} \\
0, & \text { otherwise }
\end{array}\right.
$$

In the current morningness assessment process, the following TFNs, as described in Table 1, are used corresponding to different imprecise expressions to represent imprecise opinion on the parameters of the proposed model. The remaining part of the sub-section demonstrates the process of morningness assessment corresponding to the responses of an individual.

Let $F_{X_{1}}, F_{X_{2}}, F_{X_{3}}, F_{X_{4}}$ and $F_{X_{5}}$ be five TFNs derived from the responses of an individual corresponding to five input parameters $X_{1}, X_{2}, X_{3}, X_{4}$ and $X_{5}$. Then the corresponding MFs of those TFNs can be given by $\mu_{F_{X_{1}}}\left(u_{1}\right), \mu_{F_{X_{2}}}\left(u_{2}\right), \mu_{F_{X_{3}}}\left(u_{3}\right), \mu_{F_{X_{4}}}\left(u_{4}\right)$ and $\mu_{F_{X_{5}}}\left(u_{5}\right)$ where $u_{1}, u_{2}, u_{3}, u_{4}$ and $u_{5}$ are variables in the universe of discourses $U_{1}, U_{2}, U_{3}, U_{4}$ and $U_{5}$ corresponding to $X_{1}, X_{2}, X_{3}, X_{4}$ and $X_{5}$. 
Table 1. Linguistic terms with their associated TFNs.

\begin{tabular}{|c|c|c|c|}
\hline Inexact expressions & $\begin{array}{l}\text { Fuzzy } \\
\text { Inputs }\end{array}$ & Input types & TFNs \\
\hline "...is a" & $a$ & A numerical value & $\langle a, a, a, a\rangle$ \\
\hline "...between a and b" & {$[a, b]$} & A range of number & $\langle a,(a+b) / 2,(a+b) / 2, b\rangle$ \\
\hline "...is between a and $\mathrm{c}$ and most likely to be $\mathrm{b} "$ & $\langle a, b, c\rangle$ & Triangular fuzzy numbers & $\langle a, b, b, c\rangle$ \\
\hline "...is between a and $d$ and most likely between $b$ and $c "$ & $\langle a, b, c, d\rangle$ & Trapezoidal fuzzy numbers & $\langle a, b, c, d\rangle$ \\
\hline "...is Odd" & $\langle a, b, c, d\rangle$ & A linguistic term & Odd MF $\langle a, b, c, d\rangle$ \\
\hline
\end{tabular}

FRA evaluation process on the basis of Mamdani method $^{28}$ has been employed here to determine relevant rules in the current situation for mapping the input parameters $X_{1}, X_{2}, X_{3}, X_{4}$ and $X_{5}$ with the output parameter $Z$. Subsequently, the rules are used to find the membership grades of output parameter $Z$ of that individual as follows.

If the $i^{t h}$ rule $R_{i}$ is in the form of

$$
\begin{aligned}
& R_{i} \text { : if } X_{1} \text { is } \tilde{A}_{X_{1}}^{i} \text { and } X_{2} \text { is } \tilde{A}_{X_{2}}^{i} \text { and } X_{3} \text { is } \tilde{A}_{X_{3}}^{i} \\
& \quad \text { and } X_{4} \text { is } \tilde{A}_{X_{4}}^{i} \text { and } X_{5} \text { is } \tilde{A}_{X_{5}}^{i} \text {, then } Z \text { is } \tilde{A}_{Z}^{i}
\end{aligned}
$$

where $\tilde{A}_{X_{1}}^{i}, \tilde{A}_{X_{2}}^{i}, \tilde{A}_{X_{3}}^{i}, \tilde{A}_{X_{4}}^{i}, \tilde{A}_{X_{5}}^{i}$ and $\tilde{A}_{Z}^{i}$ are the respective qualitative descriptors of $X_{1}, X_{2}, X_{3}, X_{4}, X_{5}$ and $Z$ for the $i^{\text {th }}$ rule, then the firing strength $\alpha_{i}$ of the $i^{\text {th }}$ rule corresponding to the fuzzy inputs $F_{X_{1}}, F_{X_{2}}, F_{X_{3}}$, $F_{X_{4}}$ and $F_{X_{5}}$ derived from the responses of an individual is calculated using fuzzy intersection operation as

$$
\alpha_{i}=\min \left[\begin{array}{l}
\text { height }\left(\mu_{F_{X_{1}}}\left(u_{1}\right) \wedge \mu_{\tilde{A}_{X_{1}}^{i}}\left(u_{1}\right)\right), \\
\operatorname{height}\left(\mu_{F_{X_{2}}}\left(u_{2}\right) \wedge \mu_{\tilde{A}_{X_{2}}^{i}}\left(u_{2}\right)\right), \\
h e i g h t\left(\mu_{F_{X_{3}}}\left(u_{3}\right) \wedge \mu_{\tilde{A}_{X_{3}}^{i}}\left(u_{3}\right)\right), \\
h e i g h t\left(\mu_{F_{X_{4}}}\left(u_{4}\right) \wedge \mu_{\tilde{A}_{X_{4}}^{i}}\left(u_{4}\right)\right), \\
h e i g h t\left(\mu_{F_{X_{5}}}\left(u_{5}\right) \wedge \mu_{\tilde{A}_{X_{5}}^{i}}\left(u_{5}\right)\right)
\end{array}\right]
$$

where $\mu_{F_{X_{1}}}\left(u_{1}\right), \mu_{F_{X_{2}}}\left(u_{2}\right), \mu_{F_{X_{3}}}\left(u_{3}\right), \mu_{F_{X_{4}}}\left(u_{4}\right)$ and $\mu_{F_{X_{5}}}\left(u_{5}\right)$ are respective MFs corresponding to fuzzy inputs $F_{X_{1}}, F_{X_{2}}, F_{X_{3}}, F_{X_{4}}$ and $F_{X_{5}}$ associated with the responses of that individual and $\mu_{\tilde{A}_{X_{1}}^{i}}\left(u_{1}\right), \mu_{\tilde{A}_{X_{2}}^{i}}\left(u_{2}\right)$,
$\mu_{\tilde{A}_{X_{3}}^{i}}\left(u_{3}\right), \mu_{\tilde{A}_{X_{4}}^{i}}\left(u_{4}\right)$ and $\mu_{\tilde{A}_{X_{5}}^{i}}\left(u_{5}\right)$ are respective MFs corresponding to qualitative descriptors $\tilde{A}_{X_{1}}^{i}, \tilde{A}_{X_{2}}^{i}, \tilde{A}_{X_{3}}^{i}$, $\tilde{A}_{X_{4}}^{i}, \tilde{A}_{X_{5}}^{i}$ of $X_{1}, X_{2}, X_{3}, X_{4}$ and $X_{5}$ for the $i^{\text {th }}$ rule. The output of the $i^{\text {th }}$ rule is obtained as follows.

$$
\mu_{\text {OUT }}^{i}(v)=\min \left(\alpha_{i}, \mu_{\tilde{A}_{Z}^{i}}(v)\right) .
$$

where $\alpha_{i}$ is the firing strength of the $i^{\text {th }}$ rule, $\mu_{\tilde{A}_{Z}^{i}}(v)$ represents the MF, written in terms of the variable $v$ within the universe of discourse $V$ of $Z$, corresponding to the qualitative descriptor of the output parameter $Z$ associated with consequent of the $i^{\text {th }}$ rule. $\mu_{\text {OUT }}^{i}(v)$ represents the MF of fuzzy output corresponding to the $i^{\text {th }}$ rule.

The aggregated fuzzy output $(A F O)$ corresponding to the responses of that individual is calculated using fuzzy union operation as

$$
\mu_{A F O}(v)=V_{i=1}^{n} \mu_{O U T}^{i}(v) .
$$

where $\mu_{A F O}(v)$ represents the MF of the $A F O$ in the universe of discourse $V$ of $Z$ for that particular individual. The determination of $A F O$ by the proposed FRA is shown in Fig 1.

The $A F O$ is then defuzzified to a crisp number $z^{*}$ that represents the morningness score corresponding to that particular individual using the centroid of area method as follows.

$$
Z^{*}=\frac{\sum_{j=1}^{S} \mu_{A F O}\left(v_{j}\right) \cdot v_{j}}{\sum_{j=1}^{S} \mu_{A F O}\left(v_{j}\right)} .
$$

where $v_{j} \in V(j=1,2, \cdots, s)$ are ' $s$ ' quantization of $V, \mu_{A F O}\left(v_{j}\right)$ are their membership grades corresponding to the fuzzy set $A F O$ in the universe of discourse $V$ of $Z$ and $z^{*}$ is the crisp value of $Z$ for that individual. 


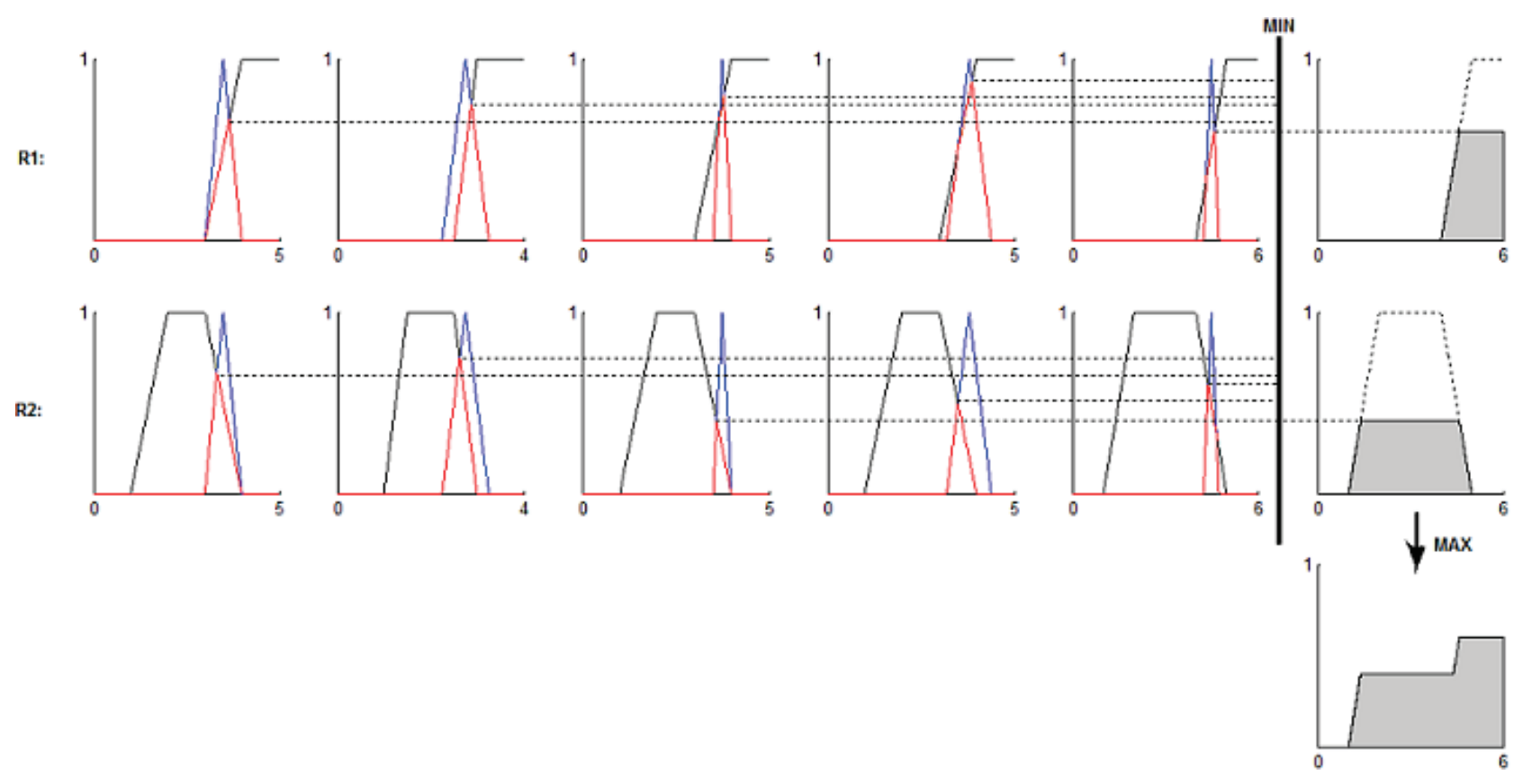

Fig. 1. Pictorial description of fuzzy input, antecedent and consequent operations for two rules.

3. Morningness assessment of a group of students in the University of Kalyani: A case study

An illustrative case example on morningness assessment of a group of students of an Indian University, viz., University of Kalyani, Kalyani, West Bengal, India has been considered to demonstrate the proposed morningness assessment methodology. Five input parameters $\left(X_{1}, X_{2}, X_{3}, X_{4}\right.$ and $\left.X_{5}\right)$ as defined in subsection 2.2 are taken into account. The outputs $(Z)$ are measured on a scale ranging between 0 to 6 . The morningness of an individual is categorized into three chronotypes, viz., 'Morning type $(M)$ ', 'Intermediate type (I)' and 'Evening type $(E)$ '.

The qualitative descriptors of five input and one output parameters have been developed for the group of students of the said University and the FRA is employed to estimate the output of each individual in terms of input parameters. The first input parameter $\left(X_{1}\right)$ describes the rising time of an individual required for his/her own feeling best rhythm and is measured on a scale ranging from 0 to 5 . Higher score implies earlier rising time of an individual required for his/her own feeling best rhythm. The qualitative descriptors of $X_{1}$ are defined as 'Early', 'Moderate' and 'Late' and their descriptions are presented in Table 2.

The second input parameter $\left(X_{2}\right)$ expresses the feelings within first half an hour after waken up in morning which is measured on a scale ranging between 0 to 4 . In this study, the qualitative descriptors are used as 'Good', 'fair' and 'Bad'. Table 3 shows the evaluation criteria of $X_{2}$ and the corresponding qualitative descriptors.

The third input parameter $\left(X_{3}\right)$ describes the sleeping time of an individual when he/she is free without any scheduling task throughout the day. On the basis of his/her response scoring has been given on a scale ranging between 0 to 5 . Higher score implies earlier sleeping time of an individual. The qualitative descriptors of $X_{3}$ are defined as 'Early', 'Moderate' and 'Late' and their descriptions are presented in Table 4.

The fourth input parameter $\left(X_{4}\right)$ expresses the time of the day at which an individual thinks that he/she reaches his/her feeling best peak and on the basis of his/her response scoring has been assigned on a scale ranging between 0 to 5 . Higher score implies earlier time to reach feeling best peak of an individual. The qualitative descriptors of $X_{4}$ are described as 'Early', 'Moderate' and 'Late' and their descriptions are presented in Table 5. 
The fifth input parameter $\left(X_{5}\right)$ defines the morningevening orientation that an individual assesses himself/herself on a scale ranging from 0 to 6 . In this study, individuals might often use qualitative descriptors as 'Morning Type', 'Rather Morning than Evening', 'Rather Evening than Morning', and 'Evening Type'. Table 6 shows the evaluation criteria of $X_{5}$ and the corresponding qualitative descriptors.

The qualitative descriptors of the output parameter $(Z)$ are characterized as 'Morning type (M)',
'Intermediate type (I)' and 'Evening type $(E)$ '. Their definitions are listed in Table 7. The morningness score is defined in a manner that the lowest score is 0 , whereas the highest score is 6. Similar to the input qualitative descriptors the trapezoidal MFs are used to describe the morningness score $Z$.

All the qualitative descriptors used in this case study are characterized by trapezoidal type MFs whose parameters are written with the form $(a, b, c, d)$.

Table 2. Representation of qualitative descriptors of Average Time for Feeling Best $\left(X_{1}\right)$.

\begin{tabular}{lllcc}
\hline Index & Qualitative descriptors & Description & Scores in preference scale (0-5) & $\begin{array}{l}\text { Parameters of MFs } \\
\text { (Trapezoidal) }\end{array}$ \\
\hline 1 & Early & Before 6:30 a.m. & $>3$ & $(3,4,5,5)$ \\
2 & Moderate & Between 6:30 a.m. to 8:00 a.m. & $1-3$ & $(1,2,3,4)$ \\
3 & Late & After 8:00 a.m. & $<1$ & $(0,0,1,2)$ \\
\hline
\end{tabular}

Table 3. Representation of qualitative descriptors of Feelings within First Half an Hour after waken up in the morning $\left(X_{2}\right)$.

\begin{tabular}{lllll}
\hline Index & Qualitative descriptors & Description & $\begin{array}{l}\text { Scores in } \\
\text { preference scale } \\
(0-4)\end{array}$ & $\begin{array}{l}\text { Parameters of MFs } \\
\text { (Trapezoidal) }\end{array}$ \\
\hline 1 & Good & Feels very fresh and energetic for doing something & $>3$ & $(2.5,3,4,4)$ \\
2 & Moderate & Feels only fresh enough for doing something & $1-3$ & $(1,1.5,2.5,3)$ \\
3 & Bad & Feels lazy for doing something. & $<1$ & $(0,0,1,1.5)$ \\
\hline
\end{tabular}

Table 4. Representation of qualitative descriptors of Sleeping Time $\left(X_{3}\right)$.

\begin{tabular}{lllll}
\hline Index & Qualitative descriptors & Description & $\begin{array}{l}\text { Scores in preference } \\
\text { scale }(0-5)\end{array}$ & $\begin{array}{l}\text { Parameters of MFs } \\
\text { (Trapezoidal) }\end{array}$ \\
\hline 1 & Early & Before 10:30 p.m. & $>3$ & $(3,4,5,5)$ \\
2 & Moderate & Between 10:30 p.m. to 2:00 a.m. & $1-3$ & $(1,2,3,4)$ \\
3 & Late & After 2:00 a.m. & $<1$ & $(0,0,1,2)$ \\
\hline
\end{tabular}

Table 5. Representation of qualitative descriptors of Feeling Best Time of a Day $\left(X_{4}\right)$.

\begin{tabular}{lllll}
\hline Index & Qualitative descriptors & Description & $\begin{array}{l}\text { Scores in } \\
\text { preference scale }\end{array}$ & $\begin{array}{l}\text { Parameters of MFs } \\
\text { (Trapezoidal) }\end{array}$ \\
\hline 1 & Early & Before 12:30 p.m. & $>3$ & $(3,4,5,5)$ \\
2 & Moderate & Between 12:30 p.m. to 10:30 p.m. & $1-3$ & $(1,2,3,4)$ \\
3 & Late & After 10:30 p.m. & $<1$ & $(0,0,1,2)$ \\
\hline
\end{tabular}

Table 6. Representation of qualitative descriptors of Self-Assessment about Morningness $\left(X_{5}\right)$.

\begin{tabular}{lllll}
\hline Index & Qualitative descriptors & Description & $\begin{array}{l}\text { Scores in } \\
\text { preference scale }\end{array}$ & $\begin{array}{l}\text { Parameters of MFs } \\
\text { (Trapezoidal) }\end{array}$ \\
\hline 1 & Morning type & Self-assessment is definitely morning type & $>4$ & $(4,5,6,6)$ \\
2 & Neither Morning nor Evening & Self-assessment is between morning and evening & $2-4$ & $(1,2,4,5)$ \\
3 & Evening type & Self-assessment is definitely evening type & $<2$ & $(0,0,1,2)$ \\
\hline
\end{tabular}


Table 7. Representation of qualitative descriptors of Morningness (Z).

\begin{tabular}{llll}
\hline Index & Qualitative descriptors & Description & $\begin{array}{l}\text { Parameters of MFs } \\
\text { (Trapezoidal) }\end{array}$ \\
\hline 1 & Morning type & Morningness score is high & $(4,5,6,6)$ \\
2 & Intermediate & Morningness score is medium & $(1,2,4,5)$ \\
3 & Evening type & Morningness score is low & $(0,0,1,2)$ \\
\hline
\end{tabular}

Since, the five input parameters are used to determine the morningness score of individual, the rule base is consisting of 243 if-then rules for this study. Table 8 depicts those rules inform 27 rule matrices in which each matrix consists of 9 rules which are generated by keeping the index of the qualitative descriptors of $X_{3}, X_{4}$ and $X_{5}$ fixed and varying those of $X_{1}$ and $X_{2}$ in between 1 and 3 .

For example, the first rule corresponding to the first matrix has been generated as follows:

IF $X_{1}=1$ (i.e., Early) and $X_{2}=1$ (i.e., Good) and $X_{3}=1$ (i,e., Early) and $X_{4}=1$ (i.e., Early) and $X_{5}=1$ (i.e., Morning type), then $Z$ is Morning Type.

Similarly, other rules are generated by considering all possible combinations.

Table 9 reflects the responses of 10 individuals corresponding to five input parameters $X_{1}, X_{2}, X_{3}, X_{4}$ and $X_{5}$. Responses of those individuals are then measured on preference scale on the basis of experts' knowledge. Since, the scores given by the experts are expressed using linguistic terms, the scores are then converted into corresponding TFNs as defined in Table 1. Based on the derived TFNs and the qualitative descriptors, the firing strength of each rule is then calculated using Eq. (3). The fuzzy implication is applied to obtain resulting fuzzy sets of the fired rules. The MFs of those output fuzzy sets corresponding to the fired rules are obtained using Eq. (4). Those MFs are then aggregated using Eq. (5) to form a single fuzzy set which represents the fuzzy output of Morningness. Finally, on the basis of the aggregated fuzzy set, the defuzzified value is calculated using Eq. (6).

To illustrate the FRA process of Morningness assessment, the calculation for the first individual has been discussed elaborately below.

The responses given by the first individual corresponding to $X_{1}, X_{2}, X_{3}, X_{4}$ and $X_{5}$ are Between 5:00 a.m. and 5:30 a.m., Very refreshed, Around 9:30 p.m., Between 7:00 a.m. and 9:00 a.m., Self-assessment is morning, respectively. These responses are measured on fuzzy preference scale based on experts' knowledge as
Between 3.85 and 4.25, Between 3 and 4, Almost 4, Between 3.95 and 4.45, and Almost 4, respectively.

The given scores are then converted into TFNs using Table 1 and are obtained as $F_{X_{1}}=\langle 3.85,4.05,4.05,4.25\rangle$, $F_{X_{2}}=\langle 3,3.5,3.5,4\rangle, \quad F_{X_{3}}=\langle 3.75 .4,4,4.25\rangle, \quad F_{X_{4}}=$ $\langle 3.95,4.2,4.2,4.45\rangle$ and $F_{X_{5}}=\langle 5.5,5.75,6,6\rangle$, respectively.

When these TFNs are injected in the proposed FRA model, only following 8 rules of the rule base are to be significant for this response.

$R_{1}$ : IF $X_{1}=1$ (i.e., Early) and $X_{2}=1$ (i.e., Good) and $X_{3}=1$ (i,e., Early) and $X_{4}=1$ (i.e., Early) and $X_{5}=1$ (i.e., Morning type), then $Z$ is Morning Type.

$R_{2}$ : IF $X_{1}=2$ (i.e., Moderate) and $X_{2}=1$ (i.e., Good) and $X_{3}=1$ (i,e., Early) and $X_{4}=1$ (i.e., Early) and $X_{5}=1$ (i.e., Morning type), then $Z$ is Morning Type.

$R_{10}$ : IF $X_{1}=1$ (i.e., Early) and $X_{2}=1$ (i.e., Good) and $X_{3}=2$ (i,e., Moderate) and $X_{4}=1$ (i.e., Early) and $X_{5}=1$ (i.e., Morning type), then $Z$ is Morning Type.

$R_{11}$ : IF $X_{1}=2$ (i.e., Moderate) and $X_{2}=1$ (i.e., Good) and $X_{3}=2$ (i,e., Moderate) and $X_{4}=1$ (i.e., Early) and $X_{5}=1$ (i.e., Morning type), then $Z$ is Morning Type.

$R_{28}$ : IF $X_{1}=1$ (i.e., Early) and $X_{2}=1$ (i.e., Good) and $X_{3}=1$ (i,e., Early) and $X_{4}=2$ (i.e., Moderate) and $X_{5}=1$ (i.e., Morning type), then $Z$ is Morning Type.

$R_{29}$ : IF $X_{1}=2$ (i.e., Moderate) and $X_{2}=1$ (i.e., Good) and $X_{3}=1$ (i,e., Early) and $X_{4}=2$ (i.e., Moderate) and $X_{5}=1$ (i.e., Morning type), then $Z$ is Morning Type.

$R_{37}$ : IF $X_{1}=1$ (i.e., Early) and $X_{2}=1$ (i.e., Good) and $X_{3}=2$ (i,e., Moderate) and $X_{4}=2$ (i.e., Moderate) and $X_{5}=1$ (i.e., Morning type), then $Z$ is Morning Type.

$R_{38}$ : IF $X_{1}=2$ (i.e., Moderate) and $X_{2}=1$ (i.e., Good) and $X_{3}=2$ (i,e., Moderate) and $X_{4}=2$ (i.e., Moderate) and $X_{5}=1$ (i.e., Morning type), then $Z$ is Intermediate Type. 

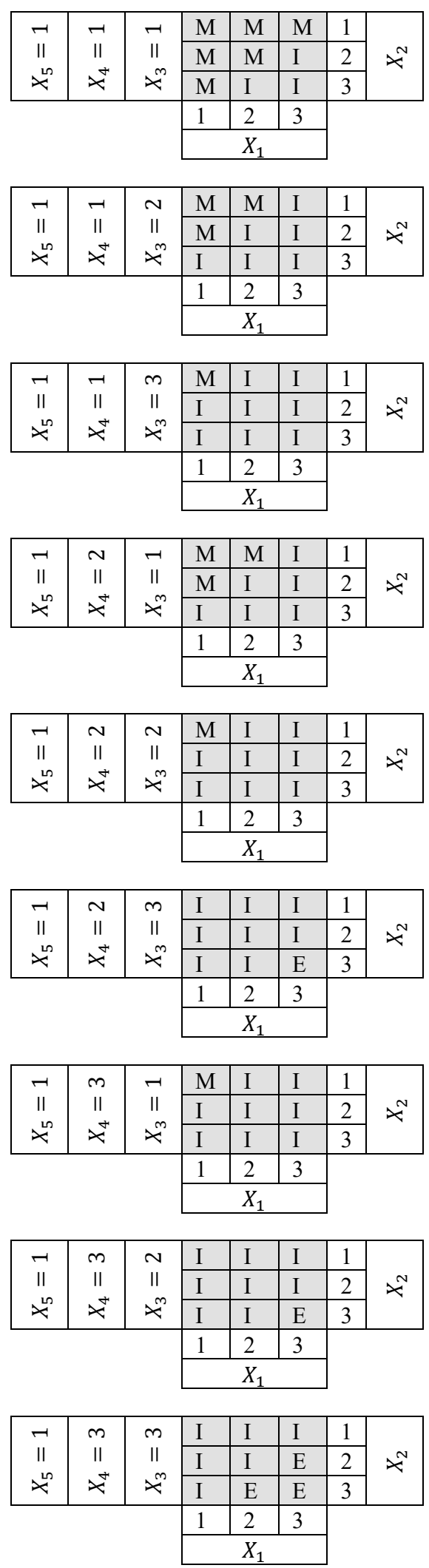

Table 8. Fuzzy rule-base matrices.
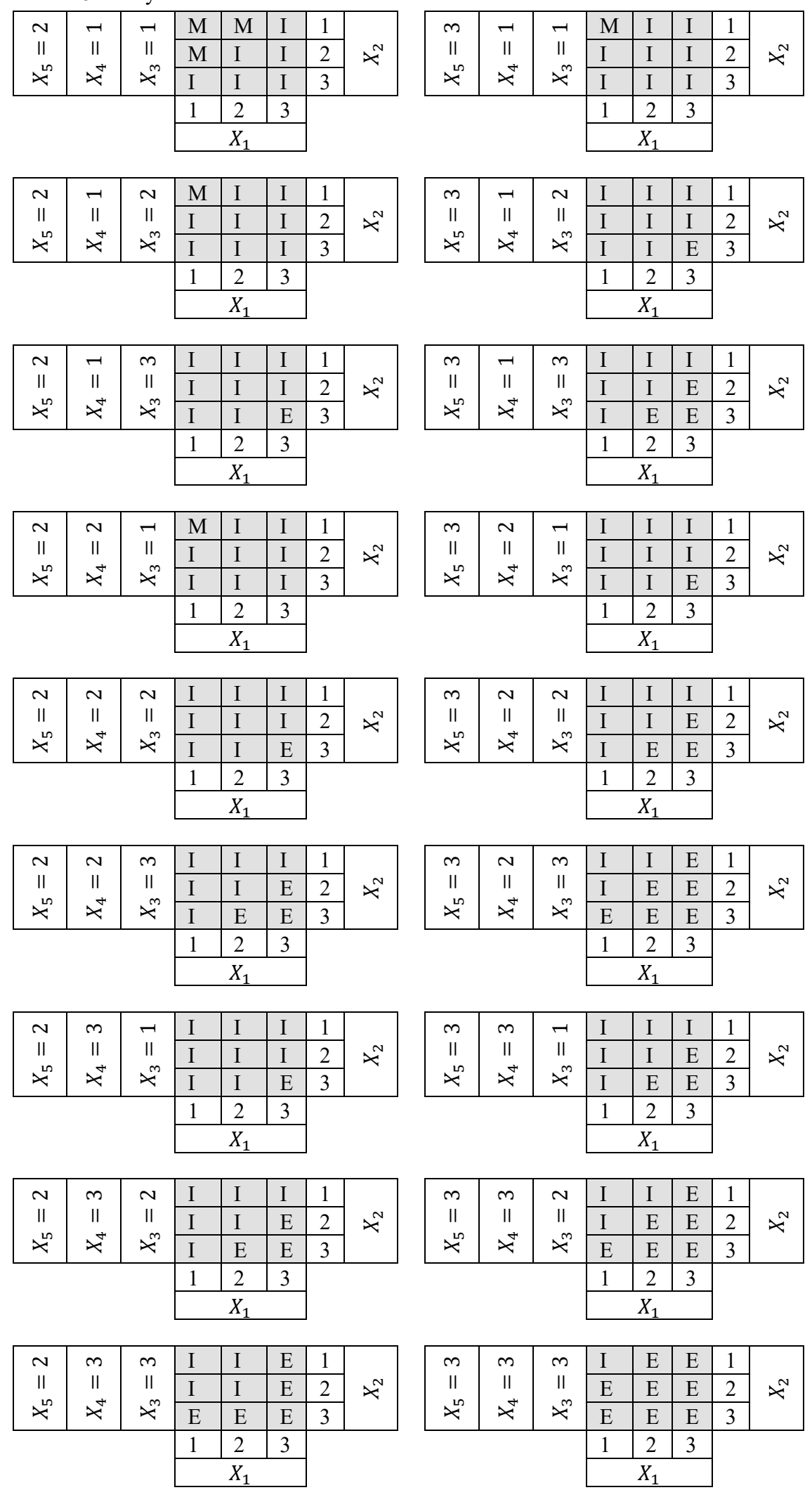

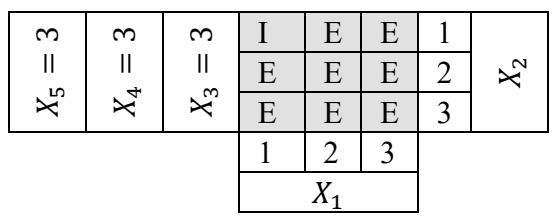


The firing strength $\alpha_{1}$ of the rule $R_{1}$ is calculated using Eq. (3) as follows

$$
\alpha_{1}=\min \left[\begin{array}{c}
\operatorname{height}\left(\mu_{F_{X_{1}}}\left(u_{1}\right) \wedge \mu_{\text {Early }}\left(u_{1}\right)\right), \\
\operatorname{height}\left(\mu_{F_{X_{2}}}\left(u_{2}\right) \wedge \mu_{\text {Good }}\left(u_{2}\right)\right), \\
\operatorname{height}\left(\mu_{F_{X_{3}}}\left(u_{3}\right) \wedge \mu_{\text {Early }}\left(u_{3}\right)\right), \\
\operatorname{height}\left(\mu_{F_{X_{4}}}\left(u_{4}\right) \wedge \mu_{\text {Early }}\left(u_{4}\right)\right), \\
\operatorname{height}\left(\mu_{F_{X_{5}}}\left(u_{5}\right) \wedge \mu_{\text {Morning type }}\left(u_{5}\right)\right) \\
=1
\end{array}\right]
$$

The MF of output fuzzy set corresponding to the rule $R_{1}$ is obtained using Eq. (4) as follows

$$
\begin{aligned}
\mu_{\text {OUT }}^{1}(v)=\min & \left(\alpha_{1}, \mu_{\text {Morning type }}(v)\right) \\
& =\min \left(1, \mu_{\text {Morning type }}(v)\right)
\end{aligned}
$$

In the same way, firing strengths and rule outputs of the remaining 7 fired rules are respectively obtained $\alpha_{2}=0.125$

$\mu_{\text {OUT }}^{2}(v)=\min \left(0.125, \mu_{\text {Morning type }}(v)\right), \alpha_{10}=0.2$ and $\quad \mu_{O U T}^{10}(v)=\min \left(0.2, \mu_{\text {Morning type }}(v)\right), \quad \alpha_{11}=$ 0.125 and $\mu_{O U T}^{11}(v)=\min \left(0.125, \mu_{\text {Morning type }}(v)\right)$, $\alpha_{28}=0.4$ and $\mu_{O U T}^{28}(v)=\min \left(0.4, \mu_{\text {Morning type }}(v)\right)$, $\alpha_{29}=0.4$ and $\mu_{\text {OUT }}^{29}(v)=\min \left(0.4, \mu_{\text {Morning type }}(v)\right)$, $\alpha_{37}=0.4$ and $\mu_{\text {OUT }}^{37}(v)=\min \left(0.4, \mu_{\text {Morning type }}(v)\right)$, and $\quad \alpha_{38}=0.4 \quad$ and $\mu_{\text {OUT }}^{38}(v)=\min \left(0.4, \mu_{\text {Intermediate type }}(v)\right)$.

The $A F O$ is then evaluated by Eq. (5) as follows:

$$
\begin{aligned}
& \mu_{A F O}(v) \\
& =\mu_{O U T}^{1}(v) \bigvee \mu_{O U T}^{2}(v) \bigvee \mu_{O U T}^{10}(v) \bigvee \mu_{O U T}^{11}(v) \\
& \bigvee \mu_{O U T}^{28}(v) \bigvee \mu_{O U T}^{29}(v) \bigvee \mu_{O U T}^{37}(v) \bigvee \mu_{O U T}^{39}(v)
\end{aligned}
$$

The $A F O$ is then defuzzified to a crisp number $z^{*}=5.0221$ by equation (6).

Similarly, the morningness scores of other 9 individuals are calculated. The results thus obtained from the responses of 10 individuals are summarized in Table 9.

\subsection{Results and discussions}

In the similar way, Morningness scores of 10 individuals are assessed and then individuals are categorized as 'Morning type $(M)$ ', 'Intermediate type
$(I)^{\prime}$ and 'Evening type (E)' by the proposed methodology as well as by existing classical technique. The proposed methodology not only categorizes the individuals but also assigns the degree of belongingness corresponding to that category. Due to fuzzy scoring technique applied to individual responses, results obtained by the proposed methodology are found to be more realistic in comparison with that of existing techniques, and also addresses some important issues like relative importance among the intra-items of the questionnaire in the study of morningness. As for example, if the responses of Individual 2 and Individual 3 are compared, then the differences that can be observed in their responses as individual 2 feels 'very refreshed' within first half an hour after waken up in the morning where as individual 3 feels 'fairly refreshed' though their rising time in the morning is same. Moreover, Individual 2 prefers to go to bed at night earlier than that of Individual 3 and reaches 'feeling best peak" earlier than that of Individual 3 which may be the indication of the fact that Individual 3 does not has that much of morning orientation compared to Individual 2. As a consequence, it is expected that they should not have the same morning-evening orientation. But, in the conventional approach the difference in morningevening orientation of Individual 2 and Individual 3 is not clearly reflected through the results since both of them are categorized as 'Morning type $(M)$ '. On the other hand, according to the proposed methodology, morningness score of Individual 2 is 4.8858 and categorized as 'Morning type $(M)^{\prime}$ ' with membership grade 0.8858 , 'Intermediate type (I)' with membership grade 0.1142 and 'Evening type $(E)$ ' with membership grade 0 , whereas morningness score of Individual 3 is 4.2366 and categorized as 'Morning type $(M)$ ' with membership grade 0.2366 , 'Intermediate type $(I)$ ' with membership grade 0.7634 and 'Evening type $(E)$ ' with membership grade 0 . Since, Individual 2 has higher belongingness in 'Morning type $(M)^{\prime}$ than that of 'Intermediate type (I)', he/she is to be treated as 'Morning type $(M)$ '. On the other hand, Individual 3 has higher belongingness in 'Intermediate type (I)' than that of 'Morning type $(M)^{\prime}$ ', he/she is to be treated as 'Intermediate type $(I)$ '. 
Table 9. Assessment of Morningness.

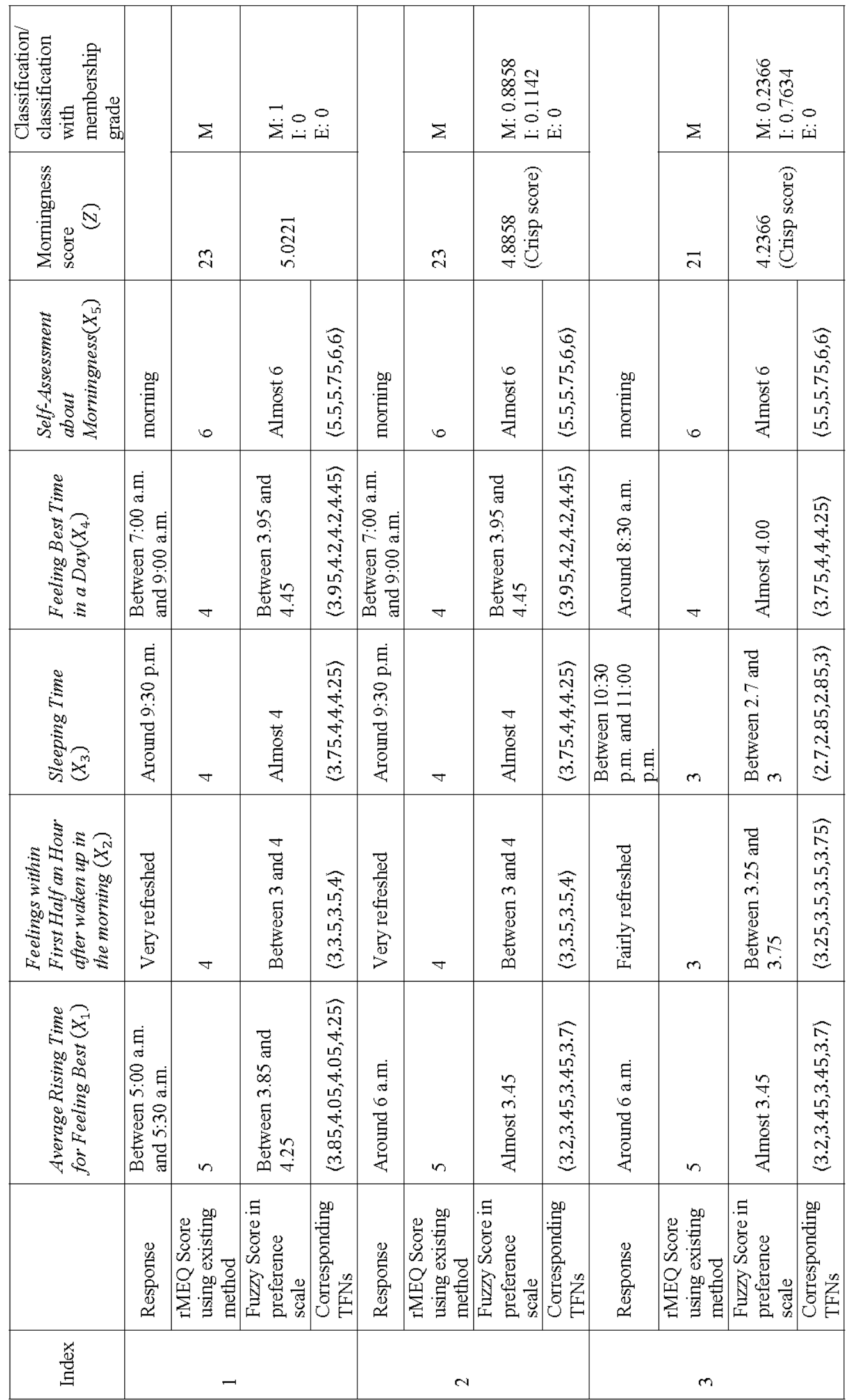


Table 9. (Continued).

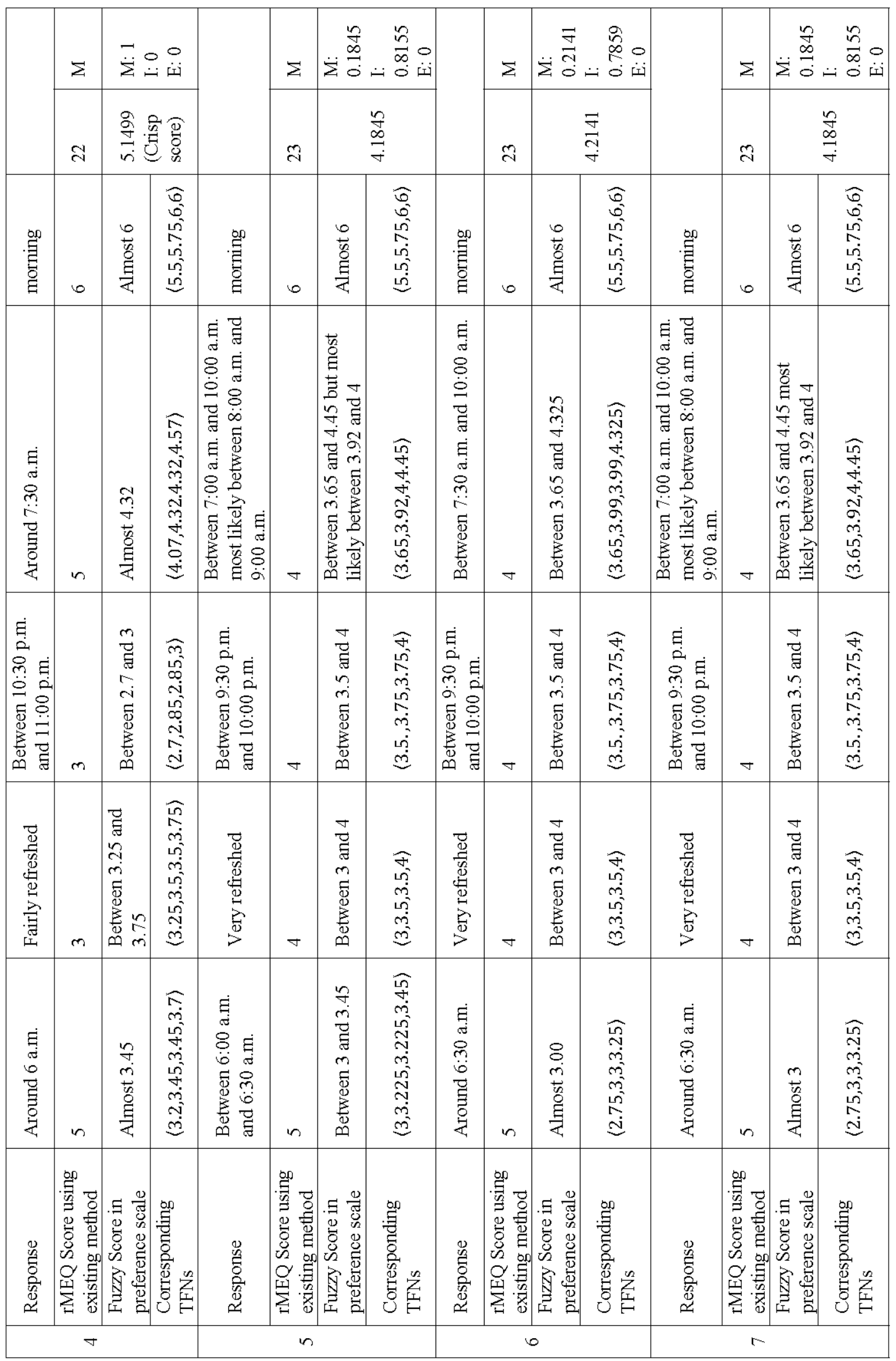


Table 9. (Continued).

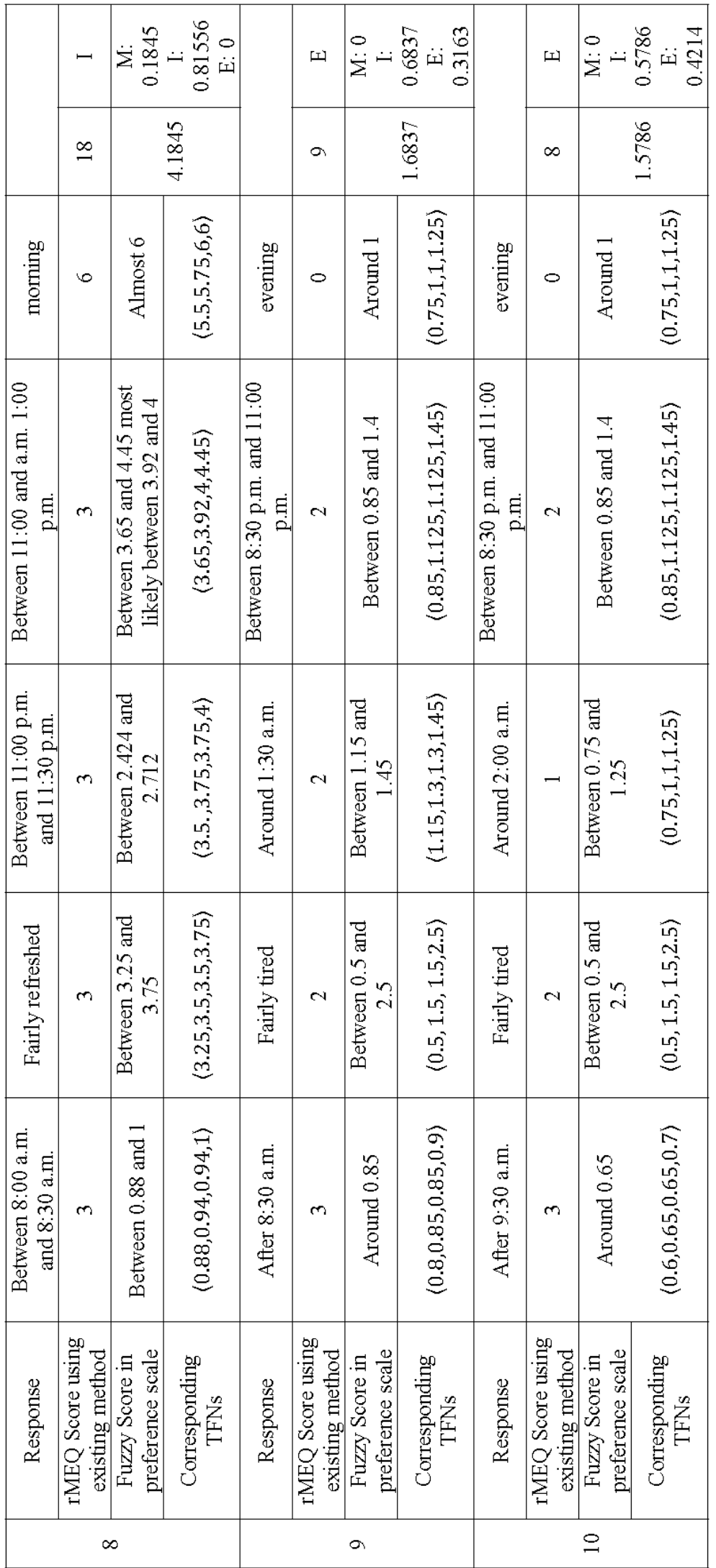


Again, if the responses of Individual 6 and Individual 7 are compared, then the only difference that can be observed is in their responses of the item Feeling Best Time in a Day $\left(X_{4}\right)$. Individual 6 reaches 'feeling best peak" between 7:30 a.m. and 10:00 a.m., whereas individual 7 reaches 'feeling best peak' between 7:00 a.m. and 10:00 a.m. and more specifically between 8:00 a.m. and 9:00 a.m. No such distinction can be made between those two Individuals by the conventional method, since, both the responses corresponding to that item got same score in preference scale. As result, according to the conventional approach both the individuals got same morningness score (same as 23) and thus both are categorized as 'Morning type $(M)$ ' which is quite unusual in real-world situation. But, according to the proposed methodology, morningness score of Individual 6 is 4.2141 and categorized as 'Morning type $(M)$ ' with membership grade 0.2141 , 'Intermediate type (I)' with membership grade 0.7859 and 'Evening type $(E)$ ' with membership grade 0; whereas morningness score of Individual 7 is 4.1845 and categorized as 'Morning type $(M)$ ' with membership grade 0.1845 , 'Intermediate type $(I)$ ' with membership grade 0.8155 and 'Evening type $(E)$ ' with membership grade 0 . Since, both individuals has higher belongingness in 'Intermediate type (I)' they are to be treated as 'Intermediate type $(I)$ ', but Individual 7 has more intermediate orientation than that of Individual 6 . This result can be justified as Individual 6 may reach 'feeling best peak' earlier than that of individual 7 and also the time duration for his/her 'feeling best peak' is much higher than that of Individual 7.

Further, if the responses of Individual 9 and Individual 10 are compared, then it can be observed that the responses corresponding to items Feelings within First Half an Hour after waken up in the morning $\left(X_{2}\right)$, Feeling Best Time in a Day $\left(X_{4}\right)$ and Self-Assessment about Morningness $\left(X_{5}\right)$ are exactly the same for both the individuals, whereas responses corresponding to other two items differs. The results obtained by the proposed method shows that there is no significant difference in their morning-evening orientation and thus both categorized as 'Intermediate type (I)' with higher belongingness than that of 'Morning type $(M)$ ' and 'Evening type $(E)$ '.

From the above comparisons, it is clear that all items of the questionnaire are not playing equally important role in the assessment process of morningness. The results obtained in Table 9 indicate that item 2 and item 4 are relatively more influential factors than the others in the rMEQ.

\section{Conclusions}

This paper presents a new systematic morningness assessment model using FRA, which is able to adopt both quantitative as well as qualitative data. Individual responses, collected through questionnaires, are measured on a preference scale using fuzzy numbers which is advantageous over the conventional crisp scoring techniques as it can capture the fuzziness of individual responses and hence provide more realistic categorization of individuals, viz., Morning type $(M)$ ', 'Intermediate type $(I)$ ' and 'Evening type $(E)$ ', along with their degree of belongingness. As a consequence, it may become an efficient tool to the researchers and practitioners to classify individuals in more convenient way.

Comparing with the conventional method, the advantages of the proposed morningness assessment methodology can be summarized as follows:

(i) It can handle impreciseness or vagueness associated in quantifying the responses through preference scale, even if the responses are given in natural languages;

(ii) The morningness can be evaluated directly through linguistic expressions which are employed in the morningness assessment;

(iii) The morningness can be assessed effectively by using expert's knowledge on different complex concepts like very tired", "fairly tired", "rather morning than evening", whose interpretation are not straightforward

(iv) It provides a more comprehensible but flexible structure for combining the information gathered through questionnaire in the context of morningness assessment.

(v) Finally, the free format form of questionnaire used in the present study provides the better way to express more realistic responses of the individuals.

Moreover, the proposed morningness assessment methodology may provide a good platform for in depth study of relative importance of the different items of several types of questionnaires which usually used in morningness study and could be a future scope for research. However, future work may include development of adaptive learning systems that can 
update the knowledge base from data when no expert knowledge is available.

\section{Acknowledgements}

The authors are thankful to the anonymous reviewers for their valuable comments and suggestions in improving the quality of the manuscript. The authors acknowledge all the university students who volunteered in this study. This work is supported by DST-PURSE Programme of the University of Kalyani, India through partial financial assistance.

\section{References}

1. L. A. Zadeh, Fuzzy logic and approximate reasoning, Synthese 30 (1975) 407-428.

2. L. A. Zadeh, A fuzzy-algorithmic approach to the definition of complex or imprecise concepts, International Journal of Man-Machine Studies 8 (1976) 249-291.

3. L. A. Zadeh, Is there a need for fuzzy logic?, Information Sciences 178 (2008) 2751-2779.

4. S. C. Yu and B. Wu, Fuzzy item response model: A new approach to generate membership function to score psychological measurement, Quality \& Quantity 43(3) (2009) 381-390.

5. C. S. Hsieh, Y. W. Chen, C. H. Wu and T. Huang, Characteristics of fuzzy synthetic decision methods for measuring student achievement, Quality \& Quantity 46(2) (2012) 523-543.

6. M. A. Gil, M. A. Lubiano, S. de la Rosa de Saa and B. ' Sinova, Analyzing data from fuzzy rating scale-based questionnaire. A case study, Psicothema 27(2) (2015) 182-191.

7. S. de la Rosa de Sáa, M. Á Gil, G. González-Rodríguez, M. T. López and M. A. Lubiano, Fuzzy rating scalebased questionnaires and their statistical analysis, IEEE Transactions on Fuzzy Systems 23(1) (2015) 111-126.

8. P. Quirós, J. M. Alonso and D. P. Pancho, Descriptive and Comparative Analysis of Human Perceptions expressed through Fuzzy Rating Scale-based Questionnaires, International Journal of Computational Intelligence Systems 9(3) (2016) 450-467.

9. F. Levi and U. Schibler, Circadian rhythms: mechanisms and therapeutic implications, Annual Review of Pharmacology and Toxicology 47(2007) 593-628.

10. A. Adan, V. Natale and H. Caci. Cognitive strategies and circadian typology, in: Progress in circadian rhythms research, ed. A. L. Léglise (Nova Science Publishers, New York, 2008), pp. 141-161.

11. A. Adan, J. Lachica, H. Caci and V. Natale, Circadian typology and temperament and character personality dimensions, Chronobiology International 27(2010) 181193.
12. T. A. Cappaert, Review: Time of Day Effect on Athletic Performance: An Update, Journal of Strength and Conditioning Research 13(4) (1999) 412-421.

13. D. J. Taylor, K. C. Clay, A. D. Bramoweth, K. Sethi and B. M. Roane, Circadian phase preference in college students: relationship with psychological functioning and academics, Chronobiology International 28 (2011) 541547.

14. I. Saksvik, B. Bjorvatn, H. Hetland, G. M. Sandal and S.Pallesen, Individual differences in tolerance to shift work - a systematic review, Sleep Medicine Reviews 15 (2010) 221-235.

15. C. A. Czeisler, J. S. Allan, S. H. Strogatz, J. M. Ronda, R. Sanchez, C. D. Rios, W. O. Freitag, G. S. Richardson and R. E. Kronauer, Bright light resets the human circadian pacemaker independent of the timing of the sleep-wake cycle, Science 233 (1986) 667-671.

16. J. A. Horne and O. Ostberg, A self-assessment questionnaire to determine morningness, eveningness, International Journal of Chronobiology, 4(1976) 97-110.

17. S. Folkard, T. H. Monk and M. C. Lobban, Toward a predictive test of adjustment to shift work, Ergonomics 22 (1979) 79-91.

18. C. S. Smith, C. Reilly and K. Midkiff, Evaluation of three circadian rhythm questionnaires with suggestions for an improved measure of morningness, Journal of Applied Psychology 74 (1989) 728-738.

19. A. Adan and H. Almirall, Horne \& Ostberg MorningnessEveningness Questionnaire: a reduced scale, Personality and Individual Differences 12 (1991) 241-53.

20. H. Caci, O. Deschaux, A. Adan and V. Natale, Comparing three morningness scales: age and gender effects, structure and cutoff criteria, Sleep Medicine $\mathbf{1 0}$ (2009) 240-245.

21. D. Milia, A. Adan, V. Natale, and C. Randler, Reviewing the psychometric properties of contemporary circadian typology measures, Chronobiology International 30(10) (2013) 1261-1271.

22. A. Biswas, D. Majumder and S. Sahu, Assessing morningness of a group of people by using fuzzy expert system and adaptive neuro fuzzy inference model, Communications in Computer and Information Science 140 (2011) 47-56.

23. A. Biswas, A. Adan, P. Haldar, D. Majumder, V. Natale, C. Randler, L. Tonetti and S. Sahu, Exploration of transcultural properties of the reduced version of the Morningness-Eveningness Questionnaire (rMEQ) using adaptive neuro fuzzy inference system, Biological Rhythm Research 45(6) (2014) 955-968.

24. A. Biswas and D. Majumder, Genetic algorithm based hybrid fuzzy system for assessing morningness, Advances in Fuzzy Systems 2014 (2014) 1-9.

25. W. Pedrycz, Human certainty in computing with fuzzy sets: an interpretability quest for higher order granular constructs, Journal of Ambient Intelligence and Humanized Computing 1 (2010) 65-74. 
26. J. M. Mendel, Computing with words and its relationships with fuzzistics, Information Sciences $\mathbf{1 7 7}$ (2007) 988-1006.

27. C. Huang and C. Moraga, Extracting fuzzy if-then rules by using the information matrix technique, Journal of Computer and System Sciences 70(1) (2005) 26-52.
28. M. An, Y. Chen and C. J. Baker, A fuzzy reasoning and fuzzy-analytical hierarchy process based approach to the process of railway risk information: A railway risk management system, Information Sciences 181 (2011) 3946-3966. 